\title{
Impact of diabetes mellitus on the early-phase arterial healing after drug-eluting stent implantation
}

\author{
Takayuki Ishihara ${ }^{1 *} \mathbb{D}$, Yohei Sotomi², Takuya Tsujimura' ${ }^{1}$ Osamu lida ${ }^{1}$, Tomoaki Kobayashi ${ }^{3}$, Yuma Hamanaka ${ }^{3}$,
} Takashi Omatsu ${ }^{3}$ Yasushi Sakata², Yoshiharu Higuchi ${ }^{3}$ and Toshiaki Mano

\begin{abstract}
Background: Early arterial healing after drug-eluting stent (DES) implantation may enable short dual-antiplatelet therapy (DAPT) strategy. The impact of diabetes mellitus (DM) on this healing has not been elucidated. We used coronary angioscopy (CAS) to compare intravascular status of DM and non-DM patients in the early phase after DES implantation.

Methods: This study was a multicenter retrospective observational study. We analyzed CAS findings of 337 lesions from 270 patients evaluated 3-5 months after DES implantation. We divided the lesion into two groups: DM $(n=149)$ and non-DM $(n=188)$. We assessed neointimal coverage $(\mathrm{NIC})$ grades (dominant, maximum and minimum), thrombus adhesion and maximum yellow color grade. NIC was graded as follows: grade 0 , stent struts were not covered; grade 1 , stent struts were covered by thin layer; grade 2, stent struts were buried under neointima. Yellow color was graded as grade 0 , white; grade 1, light yellow; grade 2, yellow; grade 3, intensive yellow.

Results: Minimum NIC grade was significantly lower in DM than in non-DM groups ( $p=0.002)$, whereas dominant and maximum NIC grades were similar between them ( $p=0.59$ and $p=0.94$, respectively), as were thrombus adhesion ( $44.3 \%$ vs. $38.8 \%, p=0.32)$ and maximum yellow color grade $(p=0.78)$. A multivariate analysis demonstrated that DM was an independent predictor of minimum NIC of grade 0 (odds ratio: 2.14, 95\% confidence interval: 1.19-3.86, $p=0.011$.

Conclusions: DM patients showed more uncovered struts than non-DM patients 3-5 months after DES implantation, suggesting that the recent ultra-short DAPT strategy might not be easily applied to DM patients.
\end{abstract}

Keywords: Coronary angioscopy, Diabetes mellitus, Drug-eluting stent

\section{Background}

Diabetes mellitus (DM) is one of the most considerable risk factors for major adverse cardiac and cerebrovascular events [1]. Patients with coronary artery disease complicated with DM often have complex lesions, and the incidences of peri-operative and long-term adverse events

*Correspondence: t.ishihara31@gmail.com

${ }^{1}$ Kansai Rosai Hospital Cardiovascular Center, 3-1-69 Inabaso, Amagasaki 660-8511, Hyogo, Japan

Full list of author information is available at the end of the article are relatively high [2]. Features of DM, particularly hyperglycemia, free fatty acids, and insulin resistance, provoke molecular mechanisms that alter the function and structure of blood vessels, including increased oxidative stress, disturbances of intracellular signal transduction, and activation of the receptor for advanced glycation end products [3]. Consequently, there is a decreased availability of nitric oxide, an increased production of endothelin, the activation of transcription factors such as NF- $\mathrm{kB}$ and AP-1, and an increased production of pro-thrombotic factors such as tissue factor and plasminogen activator 
inhibitor-1 [3]. These abnormalities contribute to the cellular events such as vasoconstriction, inflammation, and thrombosis that cause atherosclerosis and subsequently increase the risk of the adverse cardiovascular events in individuals with DM [3].

Percutaneous coronary intervention (PCI) using a drug-eluting stent (DES) has been widely applied for patients with coronary artery disease, and dual-antiplatelet therapy (DAPT) is performed in order to prevent stent thrombosis. Although the duration of DAPT after stent implantation has become shorter, it is necessary to consider the risks of both bleeding risk and thrombosis based on the patient's background $[4,5]$. The assessment of arterial healing after DES implantation is also important as it contributes to the decision whether to switch DAPT to single-antiplatelet therapy (SAPT). Early arterial healing may enable a short-DAPT strategy.

Among the intravascular imaging methods that can be used to evaluate arterial healing in vivo, coronary angioscopy (CAS) is the only method that enables the observation of the patient's intravascular status with direct and full-color vision [6-15]. The impact of DM on the safety of switching from DAPT to SAPT in the early phase has not been elucidated, and there has been no investigation of the impact of DM on the patients' early-phase arterial healing. We used CAS in the present study to determine the effects of DM on early-phase arterial healing after the implantation of a DES.

\section{Methods \\ Patients}

This was a multicenter, retrospective, observational study. From the database of CAS evaluations at each hospital, we extracted 337 lesions from 270 patients for which a CAS evaluation was performed 3-5 months after DES implantation. All DESs were implanted in de novo lesions in native coronary arteries. Basically, this study included an all-comer population. However, although angioscopic evaluation at follow-up angiography as well as staged PCI for other lesions was recommended for all patients, this was not performed when informed consent could not be obtained, or when a specialist for angioscopic evaluation was not available. The elective patients received ticlopidine (200 mg/day), clopidogrel $(75 \mathrm{mg} /$ day), or prasugrel $(3.75 \mathrm{mg} /$ day) in addition to aspirin $(100 \mathrm{mg} /$ day $)$ at least 1 week before PCI. For the emergent patients, the antiplatelet drugs (aspirin at $200 \mathrm{mg}$ and clopidogrel $300 \mathrm{mg}$ or prasugrel $20 \mathrm{mg}$ ) were loaded before PCI. The Medical Ethics Committees of Osaka Police Hospital and Kansai Rosai Hospital approved this study, and all patients provided written informed consent to participate.

\section{Angioscopic follow-up}

CAS was performed after the administrations of unfractionated heparin $(5,000 \mathrm{IU})$ into the radial or femoral artery via the inserted sheath, and isosorbide dinitrate into the coronary artery. At Osaka Police Hospital, a nonocclusion angioscopy device named VISIBLE (FiberTech Co., Ltd., Tokyo, Japan), was used. Angioscopic observation of the stented lesions was carried out while blood was cleared away from the viewing area by the injection of 3\% dextran-408 [6]. At Kansai Rosai Hospital, CAS was subsequently performed as previously described using a Fullview NEO angioscopic catheter (FiberTech) during the period from January 2010 to September 2016 [7, 8]. Briefly, an optical fiber was placed at the distal segment of the coronary artery and manually pulled back from the distal edge of the stent to the proximal edge under careful angioscopic and angiographic guidance. Since October 2016, we have been using a smart-i angioscopic catheter (Surgetech Corp., Tokyo, Japan) because the Fullview NEO was discontinued. Using guide extension catheters such as GuideLiner (Japan Lifeline, Tokyo, Japan), Guidezilla (Boston Scientific, Natrick, MA, USA) and Guideplus (NIPRO, Osaka, Japan), we blocked blood flow by flushing with low molecular weight dextran. Both angioscopic images consisted of 3,000 pixels in full color and were digitally stored for off-line analysis [9]. A smarti $6 \mathrm{~K}$ angioscopic catheter (Surgetech), which can project images with 6,000 pixels, has been available since October 2018, and this catheter was used in some cases after that time.

\section{Angioscopic analysis}

Angioscopic images were analyzed for each lesion to determine (1) the dominant, maximum, and minimum degree of neointimal coverage (NIC) over the stent; (2) the yellow color grade of the stented segment; and (3) the presence of an intra-stent thrombus. Neointimal coverage over the stent was classified into three grades as previously described: grade 0 , the stent struts were not covered by neointima and were fully visible, similar to their status immediately after implantation; grade 1 , the stent struts were visible on the surface but were covered by a thin layer; grade 2, the stent struts were not visible under neointima or the stent struts were visible through the neointima but were below the level of the neointimal surface [10]. The yellow color was graded as follows: grade 0 , white; grade 1 , light yellow; grade 2 , yellow; grade 3, intensive yellow [11]. Thrombus was defined based on the criteria adopted by the European Working Group on Coronary Angioscopy [12]. As mentioned in reports from each institution, the reproducibility was as follows: (1) Osaka Police Hospital: the inter-observer and 
intra-observer reproducibility (percent agreement) values for the interpretation of angioscopic images at this institution were $95 \%$ and $95 \%$ for stent coverage, $85 \%$ and $95 \%$ for plaque color, and $90 \%$ and $100 \%$ for thrombus, respectively [13, 14]; (2) Kansai Rosai Hospital: the estimated inter- and intra-observer $\kappa$ coefficients were $84 \%$ and $95 \%$, respectively for the dominant degree of NIC over the stent, $82 \%$ and $86 \%$ for the yellow color grade of the stented segment and $93 \%$ and $100 \%$ for the presence of intra-stent thrombus [9].

\section{Quantitative coronary angiography (QCA)}

Coronary angiography was performed in at least ten projections. The view showing the most severe stenosis was selected for QCA, which was subsequently performed using a computerized angiographic analysis system (CAAS Workstation 5.11, Pie Medical Imaging, Maastricht, The Netherlands) at the same angle of projection prior to and immediately after PCI [15].

\section{Outcome measures}

DM was defined as the use of an oral agent or insulin treatment for $\mathrm{DM}$ or an $\mathrm{HbA}_{1 \mathrm{C}}$ value $\geq 6.5 \%$. We compared the CAS outcomes between the DM and non-DM patients, and we performed a multivariate analysis to identify the outcome(s) that showed a significant between-group difference. In the DM group, the relationship between the outcomes and the baseline characteristics was examined.

\section{Statistical analyses}

All results are expressed as the mean \pm SD unless otherwise stated. Continuous variables with and without homogeneity of variance were analyzed by Student's t-test and the Welch t-test, respectively. Categorical variables were analyzed with Fisher's exact test for $2 \times 2$ comparisons. For more than $2 \times 2$ comparisons, nominal variables and ordinal variables were analyzed with the Chi-squared test and the Mann-Whitney test, respectively. The multivariate analysis was performed with a logistic regression analysis. Variables in the univariate analysis with p-values $<0.1$ were selected for the multivariate analysis. Statistical significance was defined as $\mathrm{p}<0.05$. All calculations were performed using the IBM SPSS Statistics package ver. 24 (IBM Corp., Armonk, NY, USA).

\section{Results}

\section{Baseline characteristics}

The DM group was comprised of 149 lesions from 118 patients. The characteristics of the patients in the DM and non-DM groups are summarized in Table 1. There was no significant difference in characteristics between
Table 1 Patient characteristics

\begin{tabular}{|c|c|c|c|}
\hline & $\mathrm{DM}(n=118)$ & Non-DM $(n=152)$ & $p$ value \\
\hline Male, n (\%) & $101(86)$ & $127(84)$ & 0.74 \\
\hline Age, years & $67 \pm 11$ & $69 \pm 10$ & 0.11 \\
\hline Hypertensionª n (\%) & $105(89)$ & $125(82)$ & 0.17 \\
\hline Dyslipidemia ${ }^{b}, \mathrm{n}(\%)$ & $92(78)$ & $125(82)$ & 0.44 \\
\hline Smoking status, n (\%) & & & 0.11 \\
\hline Non-smoker & $52(44)$ & $81(53)$ & \\
\hline Current smoker & $30(25)$ & $41(27)$ & \\
\hline Past smoker & $36(31)$ & $30(20)$ & \\
\hline SYNTAX score & $11.2 \pm 8.8$ & $11.1 \pm 7.0$ & 0.87 \\
\hline
\end{tabular}

Data are presented as mean \pm SD or number (\%)

DM diabetes mellitus

a Receiving antihypertensive medication, systolic blood pressure $\geq 140 \mathrm{mmHg}$, or diastolic blood pressure $\geq 90 \mathrm{mmHg}$.

b Treatment with medication, total cholesterol $\geq 220 \mathrm{mg} / \mathrm{dL}$, lowdensity lipoprotein cholesterol $\geq 140 \mathrm{mg} / \mathrm{dL}$, high-density lipoprotein cholesterol $\leq 40 \mathrm{mg} / \mathrm{dL}$, or triglycerides $\geq 150 \mathrm{mg} / \mathrm{dL}$

the groups. The uses of medication at the time of PCI and at CAS evaluations are shown in Table 2. Although the usage of a statin tended to be lower in the DM group compared to the non-DM groups, there were no significant between-group differences in medication use except for the hypoglycemic agents. More than $90 \%$ of the total series of patients received DAPT with aspirin and P2Y12 inhibitor at the time of CAS evaluation. Table 3 provides the groups' laboratory data at the time points of PCI and CAS evaluation. At the time point of PCI, although the $\mathrm{HbA}_{1 \mathrm{c}}$, fasting blood sugar values, and the triglyceride level were significantly higher in the DM group, the highdensity lipoprotein value was significantly higher in the non-DM group. Total cholesterol was significantly higher in the non-DM group at the time of the CAS evaluation. Table 4 summarizes the lesion and procedural characteristics. Among the lesion characteristics, the follow-up duration was significantly shorter and the rate of acute coronary syndrome was significantly lower in the DM group compared to the non-DM group. Regarding the procedural characteristics, the minimum stent diameter was significantly smaller in the DM group than in nonDM group. Various types of DES were used in this patient series, and the type of DES was not significantly different between the DM and non-DM groups.

\section{Angioscopic findings}

The details of the patients' angioscopic findings are illustrated in Fig. 1. The minimum NIC grade was significantly lower in the DM group than in the non-DM group, but the dominant and maximum NIC grades were similar between the groups. In the DM group, 47 lesions $(32 \%)$ demonstrated the minimum NIC of 
Table 2 Medication use

\begin{tabular}{|c|c|c|c|}
\hline & $\mathrm{DM}(\mathrm{n}=149)$ & Non-DM $(n=188)$ & $\mathrm{p}$ value \\
\hline \multicolumn{4}{|l|}{ At the time of $\mathrm{PCl}$} \\
\hline Aspirin, n (\%) & $139(93)$ & $173(92)$ & 0.41 \\
\hline Clopidogrel, n (\%) & $73(49)$ & $112(60)$ & 0.061 \\
\hline Prasugrel, n (\%) & $52(35)$ & $53(28)$ & 0.20 \\
\hline Ticlopidine, n (\%) & $7(5)$ & $8(4)$ & 1.0 \\
\hline Statin, n (\%) & $80(54)$ & $92(49)$ & 0.22 \\
\hline Insulin, n (\%) & $24(16)$ & $0(0)$ & N/A \\
\hline Biguanide, n (\%) & $38(26)$ & $0(0)$ & N/A \\
\hline DPP-4 inhibitor, n (\%) & $59(40)$ & $0(0)$ & N/A \\
\hline SGLT-2 inhibitor, n (\%) & $4(3)$ & $0(0)$ & N/A \\
\hline Thiazolidine, n (\%) & $18(12)$ & $0(0)$ & $\mathrm{N} / \mathrm{A}$ \\
\hline Sulfonylurea, n (\%) & $39(26)$ & $0(0)$ & N/A \\
\hline Glinide, n (\%) & $6(4)$ & $0(0)$ & N/A \\
\hline $\begin{array}{l}\text { a-glucosidase inhibitor, } \\
\text { n (\%) }\end{array}$ & $26(17)$ & $0(0)$ & N/A \\
\hline \multicolumn{4}{|c|}{ At the time of CAS evaluation } \\
\hline Aspirin, n (\%) & $149(100)$ & $186(99)$ & 0.31 \\
\hline Clopidogrel, n (\%) & $79(53)$ & $104(55)$ & 0.74 \\
\hline Prasugrel, n (\%) & $63(42)$ & $64(34)$ & 0.14 \\
\hline Ticlopidine, n (\%) & $6(4)$ & $8(4)$ & 1.0 \\
\hline Statin, n (\%) & $102(69)$ & $142(76)$ & 0.094 \\
\hline Insulin, n (\%) & $25(17)$ & $0(0)$ & N/A \\
\hline Biguanide, n (\%) & $43(29)$ & $0(0)$ & N/A \\
\hline DPP-4 inhibitor, n (\%) & $62(42)$ & $0(0)$ & N/A \\
\hline SGLT-2 inhibitor, n (\%) & $5(3)$ & $0(0)$ & N/A \\
\hline Thiazolidine, n (\%) & $18(12)$ & $0(0)$ & N/A \\
\hline Sulfonylurea, n (\%) & $40(27)$ & $0(0)$ & N/A \\
\hline Glinide, n (\%) & $10(7)$ & $0(0)$ & $\mathrm{N} / \mathrm{A}$ \\
\hline $\begin{array}{l}\text { a-glucosidase inhibitor, } \\
\text { n (\%) }\end{array}$ & $31(21)$ & $0(0)$ & N/A \\
\hline
\end{tabular}

Data are presented as number (\%)

CAS coronary angioscopy, DM diabetes mellitus, DPP-4 dipeptidyl peptidase-4, N/A not available, SGLT-2 sodium glucose cotransporter-2

grade 0 . The maximum yellow color grade was similarly distributed between the DM and non-DM groups. Thrombus adhesion was similar between the DM and non-DM groups $(44 \%$ vs. $39 \%, \mathrm{p}=0.32)$. The multivariate analysis conducted to detect the independent predictors of the minimum NIC of grade 0 revealed that while DM (odds ratio [OR]: 1.88, 95\% confidence interval $[\mathrm{CI}] 1.14-3.10], \mathrm{p}=0.016)$, the maximum diameter of the post-dilatation balloon (OR: 0.51, 95\% CI $0.32-0.83, \mathrm{p}=0.006)$, and the maximum inflation pressure of the post-dilatation balloon (OR: 0.93, 95\% CI $0.87-0.99, \mathrm{p}=0.048$ ) were significant predictors in the univariate analysis, only DM (OR: 2.14, 95\% CI $1.19-3.86, \mathrm{p}=0.011$ ) and the maximum diameter of the post-dilatation balloon (OR: $0.51,95 \%$ CI $0.31-0.86$,
Table 3 Laboratory Data

$\operatorname{DM}(n=149) \quad$ Non-DM $(n=188) \quad p$ value

\begin{tabular}{lccc}
\hline At the time of PCl & & & \\
$\mathrm{HbA}_{1 \mathrm{C}^{\prime}} \%$ & $7.1 \pm 1.2$ & $5.8 \pm 0.5$ & $<0.001$ \\
FBS, mg/dL & $156 \pm 60$ & $110 \pm 30$ & $<0.001$ \\
Total cholesterol, mg/dL & $176 \pm 39$ & $180 \pm 38$ & 0.25 \\
Triglyceride, mg/dL & $169 \pm 109$ & $133 \pm 100$ & 0.002 \\
HDL-cholesterol, mg/dL & $44 \pm 13$ & $50 \pm 14$ & $<0.001$ \\
LDL-cholesterol, mg/dL & $103 \pm 32$ & $105 \pm 34$ & 0.58 \\
CRP, mg/dL & $0.42 \pm 1.04$ & $0.71 \pm 2.53$ & 0.17 \\
Hemoglobin, g/dL & $13.5 \pm 2.1$ & $13.7 \pm 1.7$ & 0.45 \\
Creatinine, mg/dL & $1.2 \pm 1.9$ & $1.1 \pm 1.1$ & 0.31 \\
eGFR, mL/min/m² & $65 \pm 21$ & $64 \pm 18$ & 0.39 \\
Uric acid, mg/dL & $5.6 \pm 1.5$ & $5.9 \pm 1.5$ & 0.052 \\
At the time of CAS evaluation & & & \\
HbA1c, \% & $6.8 \pm 0.8$ & $5.7 \pm 0.5$ & $<0.001$ \\
FBS, mg/dL & $146 \pm 55$ & $107 \pm 21$ & $<0.001$ \\
Total cholesterol, mg/dL & $162 \pm 35$ & $170 \pm 31$ & 0.036 \\
Triglyceride, mg/dL & $151 \pm 112$ & $141 \pm 100$ & 0.36 \\
HDL-cholesterol, mg/dL & $47 \pm 18$ & $50 \pm 13$ & 0.057 \\
LDL-cholesterol, mg/dL & $90 \pm 26$ & $92 \pm 25$ & 0.46 \\
CRP, mg/dL & $0.24 \pm 0.52$ & $0.32 \pm 1.25$ & 0.49 \\
Hemoglobin, g/dL & $13.2 \pm 1.9$ & $13.6 \pm 1.6$ & 0.036 \\
Creatinine, mg/dL & $1.5 \pm 3.8$ & $1.1 \pm 0.9$ & 0.19 \\
eGFR, mL/min/m2 & $64 \pm 18$ & $60 \pm 17$ & 0.14 \\
Uric acid, mg/dL & $5.6 \pm 1.5$ & $5.9 \pm 1.5$ & 0.073 \\
\hline & & &
\end{tabular}

Data are presented as mean $\pm S D$

CAS coronary angiography, CRP C-reactive protein, DM diabetes mellitus, eGFR estimated glomerular filtration rate, $F B S$ fasting blood sugar, $\mathrm{HbA}_{1 \mathrm{c}}$ Hemoglobin $\mathrm{A}_{1 c^{\prime}} H D L$ high-density lipoprotein, $L D L$ low-density lipoprotein

$\mathrm{p}=0.011)$ were independent predictors even after the multivariate analysis (Table 5). We assessed the independent predictors for the minimum NIC of grade 0 in the DM group (Table 6). The univariate analysis showed that the usage of sulfonylurea at the time of the CAS evaluation was significantly associated with the minimum NIC of grade 0. Even after the adjustment for the usage of a statin (OR: 2.44, 95\% CI 1.01-5.87, p=0.047) at the time of the CAS evaluation, the following were observed to be independent predictors of the minimum NIC of grade 0 : the stent implantation pressure (OR: $0.87,95 \%$ CI $0.76-0.99, \mathrm{p}=0.040)$ and low-density lipoprotein cholesterol at the time of PCI (OR: 0.984, 95\% CI $0.972-0.997, \mathrm{p}=0.018)$, and the use of sulfonylurea (OR: 3.87, 95\% CI 1.66-9.01, $\mathrm{p}=0.002$ ).

\section{Discussion}

Our findings revealed that (1) the minimum NIC grade was lower in the patients with DM compared to the non-DM patients at 3-5 months after DES implantation; (2) the dominant NIC grade, maximum NIC grade, 
Table 4 Lesion and procedural characteristics

\begin{tabular}{|c|c|c|c|}
\hline & $\mathrm{DM}(\mathrm{n}=149)$ & Non-DM $(n=188)$ & $p$ value \\
\hline Follow-up duration, days & $115 \pm 29$ & $121 \pm 25$ & 0.031 \\
\hline Evidence of ischemia, n (\%) & & & 0.006 \\
\hline Exercise tests & $6(4)$ & $12(6)$ & \\
\hline Scintigraphy & $25(17)$ & $17(9)$ & \\
\hline FFR/iFR & $12(8)$ & $4(2)$ & \\
\hline None & $106(71)$ & $155(82)$ & \\
\hline Acute coronary syndrome, n (\%) & $45(30)$ & $78(42)$ & 0.040 \\
\hline Target vessel, n (\%) & & & 0.18 \\
\hline Left anterior descend artery & $44(30)$ & $66(35)$ & \\
\hline Left circumflex artery & $64(43)$ & $85(45)$ & \\
\hline Right coronary artery & $35(23)$ & $27(15)$ & \\
\hline Left main trunk & $6(4)$ & $10(5)$ & \\
\hline Heavy calcification, n (\%) & $17(11)$ & $19(10)$ & 0.42 \\
\hline Bifurcation, n (\%) & $53(36)$ & $73(39)$ & 0.31 \\
\hline Chronic total occlusion, n (\%) & $4(3)$ & $13(7)$ & 0.063 \\
\hline \multicolumn{4}{|l|}{ ACC/AHA classification, n (\%) } \\
\hline Type A/ B1/ B2/ C & $\begin{array}{l}15(10) / 26(17) \\
/ 24(16) / 84(56)\end{array}$ & $\begin{array}{l}20(11) / 34(18) \\
/ 41(22) / 93(50)\end{array}$ & 0.53 \\
\hline \multicolumn{4}{|l|}{ Pre-dilatation balloon } \\
\hline Maximum diameter, mm & $2.63 \pm 0.53$ & $2.66 \pm 0.52$ & 0.73 \\
\hline Maximum pressure, atm & $13 \pm 4$ & $13 \pm 3$ & 0.78 \\
\hline Maximum stent diameter, mm & $3.04 \pm 0.45$ & $3.12 \pm 0.40$ & 0.069 \\
\hline Minimum stent diameter, mm & $2.92 \pm 0.43$ & $3.02 \pm 0.43$ & 0.030 \\
\hline Total stent length, mm & $31 \pm 16$ & $31 \pm 20$ & 0.86 \\
\hline Stent implantation pressure, atm & $12 \pm 3$ & $12 \pm 3$ & 0.85 \\
\hline \multicolumn{4}{|l|}{ Post-dilatation balloon } \\
\hline Maximum diameter, $\mathrm{mm}$ & $3.20 \pm 0.56$ & $3.28 \pm 0.65$ & 0.28 \\
\hline Maximum pressure, atm & $17 \pm 4$ & $16 \pm 4$ & 0.31 \\
\hline Number of stents & $1.2 \pm 0.4$ & $1.2 \pm 0.4$ & 0.91 \\
\hline Usage of intravascular imaging device, n (\%) & & & 0.19 \\
\hline Intravascular ultrasound & $118(79)$ & $162(86)$ & \\
\hline Optical coherence tomography & $28(19)$ & $22(12)$ & \\
\hline None & $3(1)$ & $4(1)$ & \\
\hline Type of DES & & & 0.21 \\
\hline Cypher & $1(1)$ & $2(1)$ & \\
\hline Taxus & $0(0)$ & $1(1)$ & \\
\hline Endeavor & $28(19)$ & $50(27)$ & \\
\hline Xience & $30(20)$ & $32(17)$ & \\
\hline Promus & $0(0)$ & $4(2)$ & \\
\hline Resolute & $35(23)$ & $38(20)$ & \\
\hline Synergy & $26(17)$ & $24(13)$ & \\
\hline Ultimaster & $21(14)$ & $23(12)$ & \\
\hline Orsiro & $0(0)$ & $3(2)$ & \\
\hline BioFreedom & $8(5)$ & $11(6)$ & \\
\hline \multicolumn{4}{|l|}{ QCA data } \\
\hline \multicolumn{4}{|l|}{ Pre-PCl } \\
\hline Minimum lumen diameter, mm & $0.95 \pm 0.57$ & $0.86 \pm 0.57$ & 0.14 \\
\hline Reference diameter, mm & $2.65 \pm 0.69$ & $2.78 \pm 0.80$ & 0.11 \\
\hline Diameter stenosis, \% & $64 \pm 20$ & $70 \pm 19$ & 0.020 \\
\hline Lesion length, mm & $15 \pm 9$ & $17 \pm 12$ & 0.13 \\
\hline
\end{tabular}


Table 4 (continued)

\begin{tabular}{llll}
\hline & DM $(\mathbf{n}=\mathbf{1 4 9})$ & Non-DM $(\mathbf{n}=188)$ & $\mathbf{p ~ v a l u e ~}$ \\
\hline Post-PCl & & & \\
Minimum lumen diameter, mm & $2.39 \pm 0.52$ & $2.52 \pm 0.51$ & 0.028 \\
Reference diameter, mm & $2.85 \pm 0.55$ & $3.03 \pm 0.59$ & 0.005 \\
Diameter stenosis, \% & $16 \pm 8$ & $17 \pm 8$ & 0.50 \\
\hline
\end{tabular}

Data are presented as mean \pm SD or number (\%)

$D E S$ drug-eluting stent, $D M$ diabetes mellitus, $F F R$ fractional flow reserve, $i F R$ instantaneous wave-free ratio, $P C I$ percutaneous coronary intervention, $Q C A$ quantitative coronary angiography

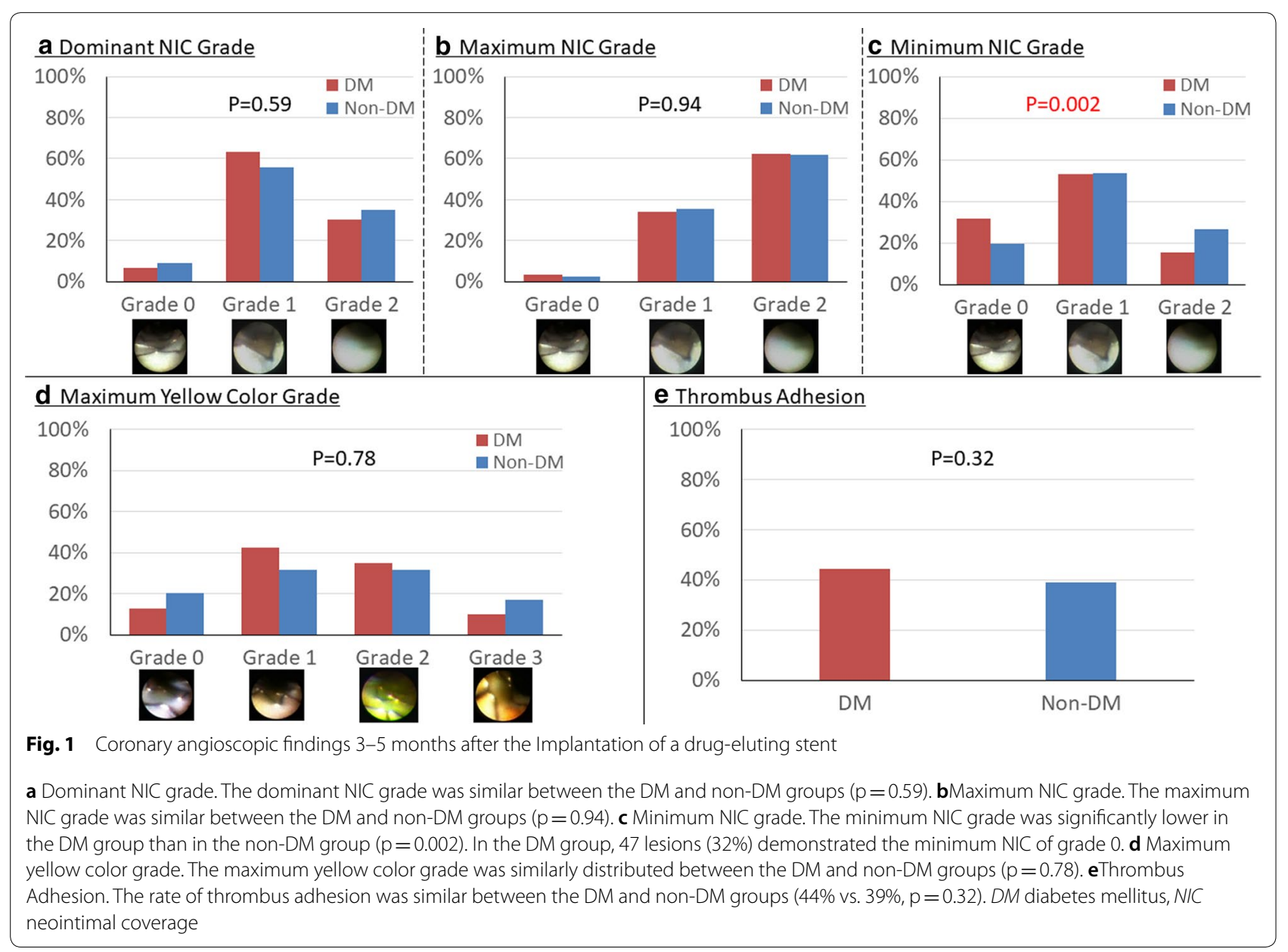

yellow color grade, and the incidence of thrombus adhesion were similar between the DM and non-DM groups; (3) DM was an independent factor for predicting the minimum NIC of grade 0 , which demonstrates uncoverage; (4) in the DM group, the use of sulfonylurea was an independent predictor of the minimum NIC of grade 0 even after the adjustment for confounding factors. To the best of our knowledge, this is the first report describing the relationship between early-phase arterial healing after DES implantation and DM.

Immediately after stent implantation in coronary arteries, bare stent struts are in direct contact with the vessel wall, and the process of arterial healing begins as follows [16, 17]: (1) the first step in arterial healing is the formation of a local thrombus. At the injury site, platelets, fibrin, and red blood cells accumulate and a local 
Table 5 Logistic regression analysis for minimum NIC of grade 0 (Uncoverage)

\begin{tabular}{|c|c|c|c|c|c|c|}
\hline & \multicolumn{3}{|c|}{ Univariate } & \multicolumn{3}{|c|}{ Multivariate } \\
\hline & OR & $95 \% \mathrm{Cl}$ & $p$ value & OR & $95 \% \mathrm{Cl}$ & $\mathrm{p}$ value \\
\hline Male & 1.39 & $0.64-2.84$ & 0.48 & & & \\
\hline $\begin{array}{l}\text { Age } \\
\text { (1 year increase) }\end{array}$ & 1.00 & $0.98-1.03$ & 0.98 & & & \\
\hline $\begin{array}{l}\text { BMl } \\
\left(1 \mathrm{~kg} / \mathrm{m}^{2} \text { increase }\right)\end{array}$ & 1.03 & $0.96-1.09$ & 0.44 & & & \\
\hline Hypertension & 0.83 & $0.42-1.63$ & 0.60 & & & \\
\hline Dyslipidemia & 1.39 & $0.73-2.65$ & 0.35 & & & \\
\hline DM & 1.88 & $1.14-3.10$ & 0.016 & 2.14 & $1.19-3.86$ & 0.011 \\
\hline Aspirin* & 0.33 & $0.02-5.32$ & 0.44 & & & \\
\hline Clopidogrel* & 0.96 & $0.59-1.58$ & 0.90 & & & \\
\hline Prasugrel* & 1.43 & $0.86-2.36$ & 0.19 & & & \\
\hline Ticlopidine* & 0.22 & $0.03-1.73$ & 0.20 & & & \\
\hline Statin* & 1.55 & $0.86-2.78$ & 0.16 & & & \\
\hline Follow-up duration & 0.995 & $0.986-1.004$ & 0.31 & & & \\
\hline ACS & 0.89 & $0.53-1.50$ & 0.70 & & & \\
\hline $\begin{array}{l}\text { Pre-dilatation balloon maximum diameter } \\
(1 \mathrm{~mm} \text { increase) }\end{array}$ & 1.11 & $0.75-1.66$ & 0.60 & & & \\
\hline $\begin{array}{l}\text { Pre-dilatation balloon maximum inflation pressure } \\
\text { ( } 1 \mathrm{~atm} \text { increase) }\end{array}$ & 1.04 & $0.97-1.12$ & 0.26 & & & \\
\hline $\begin{array}{l}\text { Maximum stent diameter } \\
(1 \mathrm{~mm} \text { increase) }\end{array}$ & 1.24 & $0.69-2.21$ & 0.48 & & & \\
\hline $\begin{array}{l}\text { Minimum stent diameter } \\
\text { (1 mm increase) }\end{array}$ & 0.90 & $0.51-1.60$ & 0.72 & & & \\
\hline $\begin{array}{l}\text { Stent implantation pressure } \\
\text { (1 atm increase) }\end{array}$ & 0.97 & $0.91-1.04$ & 0.43 & & & \\
\hline $\begin{array}{l}\text { Post-dilatation balloon maximum diameter } \\
\text { (1 mm increase) }\end{array}$ & 0.51 & $0.32-0.83$ & 0.006 & 0.51 & $0.31-0.86$ & 0.011 \\
\hline $\begin{array}{l}\text { Post-dilatation balloon maximum inflation pressure } \\
\text { ( } 1 \mathrm{~mm} \text { increase) }\end{array}$ & 0.93 & $0.87-0.990$ & 0.048 & 0.93 & $0.86-1.001$ & 0.052 \\
\hline \multicolumn{7}{|l|}{ At the time of $\mathrm{PCl}$} \\
\hline $\begin{array}{l}\text { Total cholesterol } \\
\text { (1 mg/dL increase) }\end{array}$ & 0.995 & $0.989-1.002$ & 0.18 & & & \\
\hline $\begin{array}{l}\text { Triglyceride } \\
\text { (1 mg/dL increase) }\end{array}$ & 1.000 & $0.998-1.003$ & 0.68 & & & \\
\hline $\begin{array}{l}\text { HDL-cholesterol } \\
\text { (1 mg/dL increase) }\end{array}$ & 0.995 & $0.976-1.014$ & 0.60 & & & \\
\hline $\begin{array}{l}\text { LDL-cholesterol } \\
\text { (1 mg/dL increase) }\end{array}$ & 0.994 & 0/987-1.002 & 0.15 & & & \\
\hline \multicolumn{7}{|l|}{ At the time of CAS evaluation } \\
\hline $\begin{array}{l}\text { Total cholesterol } \\
\text { ( } 1 \mathrm{mg} / \mathrm{dL} \text { increase) }\end{array}$ & 0.997 & $0.990-1.005$ & 0.47 & & & \\
\hline $\begin{array}{l}\text { Triglyceride } \\
\text { (1 mg/dL increase) }\end{array}$ & 1.001 & $0.999-1.003$ & 0.50 & & & \\
\hline $\begin{array}{l}\text { HDL-cholesterol } \\
\text { (1 mg/dL increase) }\end{array}$ & 0.992 & $0.975-1.010$ & 0.38 & & & \\
\hline $\begin{array}{l}\text { LDL-cholesterol } \\
\text { (1 mg/dL increase) }\end{array}$ & 0.997 & $0.987-1.007$ & 0.56 & & & \\
\hline
\end{tabular}

* Medication at the time of coronary angioscopic evaluation. $A C S$ acute coronary syndrome, $B M /$ body mass index, $C A S$ coronary angioscopy, DM diabetes mellitus, $H D L$ high-density lipoprotein, $L D L$ low-density lipoprotein, $P C I$ percutaneous coronary intervention 
Table 6 Logistic regression analysis for minimum NIC of Grade 0 (Uncoverage) in patients with DM

\begin{tabular}{|c|c|c|c|c|c|c|}
\hline & \multicolumn{3}{|c|}{ Univariate } & \multicolumn{3}{|c|}{ Multivariate } \\
\hline & OR & $95 \% \mathrm{Cl}$ & $p$ value & OR & $95 \% \mathrm{Cl}$ & $p$ value \\
\hline Male & 1.09 & $0.39-3.30$ & 1.00 & & & \\
\hline $\begin{array}{l}\text { Age } \\
\text { (1 year increase) }\end{array}$ & 1.008 & 098-1.04 & 0.65 & & & \\
\hline BMI & 1.04 & $0.96-1.12$ & 0.35 & & & \\
\hline Hypertension & 1.12 & $0.37-3.38$ & 1.00 & & & \\
\hline Dyslipidemia & 2.01 & $0.82-5.14$ & 0.14 & & & \\
\hline Aspirin* & - & - & - & & & \\
\hline Clopidogrel* & 1.01 & $0.51-2.02$ & 1.00 & & & \\
\hline Prasugrel* & 1.15 & $0.58-2.32$ & 0.72 & & & \\
\hline Ticlopidine* & 0.42 & $0.048-3.71$ & 0.38 & & & \\
\hline Statin* & 2.11 & $0.94-4.72$ & 0.088 & 2.44 & $1.01-5.87$ & 0.047 \\
\hline Insulin, n (\%) & 0.82 & $0.32-2.11$ & 0.82 & & & \\
\hline Biguanide, n (\%) & 1.43 & $0.68-3.03$ & 0.44 & & & \\
\hline DPP-4 inhibitor, n (\%) & 1.36 & $0.68-2.74$ & 0.48 & & & \\
\hline SGLT-2 inhibitor, n (\%) & 0.53 & $0.058-4.90$ & 0.50 & & & \\
\hline Thiazolidine, n (\%) & 0.82 & $0.27-2.44$ & 0.79 & & & \\
\hline Sulfonylurea, n (\%) & 2.62 & $1.23-5.57$ & 0.016 & 3.87 & $1.66-9.01$ & 0.002 \\
\hline Glinide, n (\%) & 0.93 & $0.23-3.75$ & 0.61 & & & \\
\hline a-glucosidase inhibitor, n (\%) & 1.78 & $0.79-4.04$ & 0.19 & & & \\
\hline $\begin{array}{l}\text { Follow-up duration } \\
\text { (1 day increase) }\end{array}$ & 0.995 & $0.984-1.007$ & 0.44 & & & \\
\hline ACS & 1.13 & $0.53-2.37$ & 0.85 & & & \\
\hline $\begin{array}{l}\text { Pre-dilatation balloon maximum diameter } \\
\text { ( } 1 \mathrm{~mm} \text { increase) }\end{array}$ & 1.09 & $0.63-1.90$ & 0.76 & & & \\
\hline $\begin{array}{l}\text { Pre-dilatation balloon maximum inflation pressure } \\
\text { (1 atm increase) }\end{array}$ & 1.06 & $0.96-1.16$ & 0.28 & & & \\
\hline $\begin{array}{l}\text { Maximum stent diameter } \\
\text { (1 } \mathrm{mm} \text { increase) }\end{array}$ & 1.49 & $0.69-3.20$ & 0.31 & & & \\
\hline $\begin{array}{l}\text { Minimum stent diameter } \\
\text { (1 mm increase) }\end{array}$ & 1.13 & $0.50-2.53$ & 0.77 & & & \\
\hline $\begin{array}{l}\text { Stent implantation pressure } \\
\text { (1 atm increase) }\end{array}$ & 0.91 & $0.81-1.02$ & 0.097 & 0.87 & $0.76-0.99$ & 0.040 \\
\hline $\begin{array}{l}\text { Post-dilatation balloon maximum diameter } \\
\text { (1 mm increase) }\end{array}$ & 0.86 & $0.43-1.73$ & 0.67 & & & \\
\hline $\begin{array}{l}\text { Post-dilatation balloon maximum inflation pressure } \\
\text { (1 mm increase) }\end{array}$ & 0.94 & $0.84-1.05$ & 0.26 & & & \\
\hline \multicolumn{7}{|l|}{ At the time of $\mathrm{PCl}$} \\
\hline $\begin{array}{l}\text { HbA1c } \\
\text { (1\% increase) }\end{array}$ & 1.07 & $0.81-1.42$ & 0.65 & & & \\
\hline $\begin{array}{l}\text { FBS } \\
(1 \mathrm{mg} / \mathrm{dL} \text { increase })\end{array}$ & 1.001 & $0.996-1.007$ & 0.67 & & & \\
\hline $\begin{array}{l}\text { CRP } \\
(1 \mathrm{mg} / \mathrm{dL} \text { increase })\end{array}$ & 1.16 & $0.84-1.61$ & 0.36 & & & \\
\hline $\begin{array}{l}\text { Total cholesterol } \\
\text { (1 mg/dL increase) }\end{array}$ & 0.996 & $0.987-1.005$ & 0.35 & & & \\
\hline $\begin{array}{l}\text { Triglyceride } \\
\text { (1 mg/dL increase) }\end{array}$ & 1.000 & $0.997-1.003$ & 0.85 & & & \\
\hline $\begin{array}{l}\text { HDL-cholesterol } \\
(1 \mathrm{mg} / \mathrm{dL} \text { increase })\end{array}$ & 1.022 & $0.994-1.050$ & 0.12 & & & \\
\hline $\begin{array}{l}\text { LDL-cholesterol } \\
\text { (1 mg/dL increase) }\end{array}$ & 0.989 & $0.978-1.000$ & 0.056 & 0.984 & $0.972-0.997$ & 0.018 \\
\hline At the time of CAS evaluation & & & & & & \\
\hline
\end{tabular}


Table 6 (continued)

\begin{tabular}{|c|c|c|c|c|}
\hline \multirow[b]{2}{*}{$\begin{array}{l}\mathrm{HbA} 1 \mathrm{c} \\
\text { (1\% increase) }\end{array}$} & \multicolumn{3}{|c|}{ Univariate } & \multirow[t]{2}{*}{ Multivariate } \\
\hline & 1.23 & $0.82-1.86$ & 0.32 & \\
\hline $\begin{array}{l}\text { FBS } \\
\text { (1 mg/dL increase) }\end{array}$ & 0.999 & $0.992-1.005$ & 0.70 & \\
\hline $\begin{array}{l}\text { CRP } \\
\text { (1 mg/dL increase) }\end{array}$ & 0.53 & $0.18-1.55$ & 0.53 & \\
\hline $\begin{array}{l}\text { Total cholesterol } \\
\text { (1 mg/dL increase) }\end{array}$ & 0.999 & 0.989-1.009 & 0.81 & \\
\hline $\begin{array}{l}\text { Triglyceride } \\
\text { (1 mg/dL increase) }\end{array}$ & 1.002 & 0.999-1.005 & 0.13 & \\
\hline $\begin{array}{l}\text { HDL-cholesterol } \\
\text { (1 mg/dL increase) }\end{array}$ & 1.001 & $0.982-1.021$ & 0.91 & \\
\hline $\begin{array}{l}\text { LDL-cholesterol } \\
\text { (1 mg/dL increase) }\end{array}$ & 0.990 & $0.977-1.004$ & 0.17 & \\
\hline
\end{tabular}

* Medication at the time of CAS evaluation. ACS acute coronary syndrome, $B M I$ body mass index, CRPC-reactive protein, $D M$ diabetes mellitus, FBS fasting blood sugar, $\mathrm{HbA}_{1 \mathrm{c}}=$ Hemoglobin $\mathrm{A}_{1 \mathrm{c}^{\prime}}, \mathrm{HDL}$ high-density lipoprotein, $L D L$ low-density lipoprotein

thrombus is formed; (2) then, inflammatory cells such as macrophages infiltrate the site; (3) inflammatory cells secrete various growth factors such as platelet-derived growth factor, and smooth muscle cells (SMCs) migrate into the site and begin to proliferate; (4) at 2 weeks after stenting, in addition to the proliferation of SMCs, the extracellular matrix is formed. Neointima formation, that is, neointimal coverage is completed in 12 weeks. The neointima is lined by one layer of endothelial cells which served as an antithrombotic barrier. However, delayed arterial healing sometimes occurs after DES implantation due to the component of DES instead of preventing SMC proliferation, which can lead to in-stent restenosis [18]. A pathological study suggested that widely uncovered struts are a risk factor for stent thrombosis [19], and optical coherence tomography (OCT) studies reported that uncoverage was one of the mechanisms of stent thrombosis [20-22]. There have been several articles which mentioned the neointimal coverage in relation to DM evaluated by OCT. Briguori $C$ et al. elucidated that baseline on-clopidogrel platelet reactivity and complex lesions were independent predictors of uncovered strut rate at 3 months [23]. Kubo $\mathrm{T}$ et al. compared the OCT findings between 1st generation sirolimus-eluting stent and 1st generation paclitaxel-eluting stent, and they demonstrated that 1st generation sirolimus-eluting stent showed stronger prohibition of neointimal hyperplasia compared with 1st generation paclitaxel-eluting stent in DM patients as well as in non-DM patients [24]. Kuroda et al. reported that large glucose fluctuations were an independent risk factor for impaired uniform vessel healing after second-generation DES [25]. However, these articles did not compare the early-phase arterial healing between DM and non-DM patients. In the present study, the CAS evaluation demonstrated that the rate of the minimum NIC of grade 0 was significantly higher in the DM group compared to the non-DM group at 3-5 months after DES implantation, which suggests that arterial healing is more delayed in patients with DM compared to those without it.

There are several reports regarding the relationship between the findings of intravascular imaging devices and DM. Kurihara et al. used angioscopy and observed that compared to non-diabetic patients, in pre-diabetic and diabetic patients the number of yellow plaques was greater and the intensity of yellow was greater [26]. They also reported that the number of yellow plaques and the maximum yellow color grade were significantly greater in patients with diabetic retinopathy than in those without it [27]. However, in the present study the yellow color grade was similar between the DM and non-DM groups. Kurihara et al. assessed the CAS findings of the native coronary arteries, whereas we evaluated them 3-5 months after DES implantation. Even with the observation in the relatively early phase after the DES implantation, the difference in the timing of the CAS observations would contribute to the difference in the yellow color grade outcome.

Our present analyses revealed that the post-dilatation balloon size and post-dilatation balloon inflation pressure were the negative predictors of the minimum NIC of grade 0. A study of peripheral arteries showed that the oversized stents caused more neointimal proliferation, which was due to the greater injury to the vessel wall [31]. In addition, malapposition was related to the subsequent incomplete NIC [27]. Adequate strut embedment may cause better neointimal coverage [27, 32, 33]. Since a smaller balloon size and lower inflation pressure 
would result in less injury to the vessel wall, the difficulty of achieving complete apposition to the vessel wall and inadequate strut embedment, the post-dilatation balloon size and post-dilatation balloon inflation pressure were negatively associated with uncoverage in this study.

Although a previous article revealed that the negative prognostic effect of DM following contemporary PCI was heightened in the presence of insulin treatment [34], insulin therapy did not impact on the early-phase arterial healing in the current study. Instead, we observed that the use of sulfonylurea was an independent predictor of the minimum NIC of grade 0 in the DM patients. It is apparent that aggressive glucose-lowering therapy increased the mortality of DM patients [35], and it has been reported that the use of sulfonylurea itself increased the risk of adverse cardiovascular events [36, 37]. Although the mechanisms underlying the relationship between the use of sulfonylurea and delayed arterial healing after DES implantation in the early phase are not yet understood, it appears that the delayed healing caused by the usage of sulfonylurea may contribute to patients' poor clinical outcomes. Our present findings also revealed that the glucose control parameters such as the $\mathrm{HbA}_{1 \mathrm{c}}$ had no association with the NIC, and the aggressive glucose control did not impact on the early-phase arterial healing after DES implantation. Although the precise mechanism remains to be undetermined, sulfonylurea treatment should be avoided to prescribe in patients with DM.

There have been several reports which mentioned the relationship between thrombogenicity and DM. Nusca et al. reported that glyco-metabolic state significantly correlated with high platelet reactivity in well-controlled type 2 DM patients on clopidogrel therapy and HbA1c identified patients at higher thrombotic risk but the highest diagnostic accuracy was achieved by combining glycemic variability and HbA1c [38]. Lee et al. mentioned that impaired glucose metabolism was associated with increased thrombin generation potential in patients undergoing PCI [39]. However, the incidence of thrombus adhesion was similar between DM and non-DM patients in the current study. This would be because angioscopic thrombus does not directly mean the thrombogenicity. In other words, angioscopic thrombus adhesion is a benchmark of the completeness of arterial healing because it does not occur where satisfactory arterial healing is achieved [40, 41].

Recent guidelines note that the patient's bleeding risk and the thrombotic risk should be considered when selecting the duration of DAPT [42-44]. The DAPT score is a landmark of the duration of DAPT performed 1 year after stent implantation, and the presence of DM is one of the factors that encourages the longer DAPT [42]. The PRESICE DAPT score, which evaluates the duration of
DAPT at the time of stent implantation, does not include DM as a factor [43]. In the PARIS scoring system, which predicts the risk of thrombotic and bleeding events after discharge based on only the patient's background, DM is one of the factors that increases the thrombotic risk [44]. A recent European Society of Cardiology guideline also suggests that diffuse lesions in an individual with $\mathrm{DM}$ is a risk for stent thrombosis [3]. A diabetic subanalysis from the PEGASUS-TIMI 54 scribes this point concluding that prolonged DAPT regimens is beneficial in patients with DM [45]. Furthermore, the DAPT score is utilized for prolonged DAPT regimens after one year [42]. Numerous recent trials also have investigated the optimal therapy time for DAPT in non-exclusive DM population below one year in which the results leaves room for debate [46-48]. The patients with DM were low in these trials and therefore these results should not be applied to diabetic patients. In addition, although some clinical trials revealed that the clinical outcomes with short DAPT were non-inferior to those with long DAPT in DM patients, the duration of short DAPT was around 6 months $[49,50]$. In the present study, the CAS evaluations demonstrated that the rate of the minimum NIC of grade 0 was significantly higher in the DM group than in the non-DM group 3-5 months after DES implantation, which is consistent with the concept that DM is a factor that increases the thrombotic risk even in the early phase. Clinicians should therefore pay attention to the possibility of switching from DAPT to SAPT in the early phase for patients with DM, and the recent ultra-short DAPT strategy might not be easily applied to DM patients.

\section{Limitations}

This study has several limitations. First, it was a nonrandomized, retrospective, observational study; however, the multi-center aspect of the study made the sample size relatively large compared to those of previous studies. Second, an angioscopically observed thrombus does not directly indicate the risk of stent thrombosis. Third, although underlying plaque morphology is associated with vessel healing with neointimal formation, we did not evaluate the baseline lesion morphology by fixed intravascular imaging devices. Forth, since there was a possibility of some differences between 3 months and 5 months after stenting in regard to the NIC, more strict selection of the cases regarding the timing of CAS evaluation would be preferable. However, since the sample size was limited in this retrospective analysis, we cannot help including patients with 3-5 months follow-up. In addition, the follow-up duration was not independently associated with minimum NIC of grade 0 as shown in Table 5 . Fifth, the CAS devices were not fixed between these facilities, because this was a retrospective study. Sixth, we 
included the various type of DES, although it would have a great impact on the results. However, since this was a retrospective study and the sample size was limited, we could not help including the various type of stent, and the type of DES was similar between DM and non-DM groups as shown in Table 4. Seventh, the follow-up time in the DM group was shorter than in the non-DM group and it could affect in the endpoints results. However, in terms of the minimum NIC of Grade 0, follow-up duration did not impact on the result as shown in Table 5 . Finally, on some occasions the CAS could not completely evaluate the entire stented segment because of the limitations of the CAS visual field, especially in angulated or tortuous lesions.

\section{Conclusions}

The minimum NIC grade was lower in patients with the DM than in those without DM at 3-5 months after DES implantation, and DM was an independent predictor of the minimum NIC of grade 0 , which demonstrates uncoverage, suggesting that the recent ultra-short DAPT strategy might not be easily applied to DM patients.

\begin{abstract}
Abbreviations
CAS: Coronary angioscopy; DAPT: Dual-antiplatelet therapy; DES: Drug-eluting stent; DM: Diabetes mellitus; NIC: Neointimal coverage; OCT: Optical coherence tomography; PCI: Percutaneous coronary intervention; SAPT: Singleantiplatelet therapy.

Acknowledgements

We wish to thank Mr. Naoya Kurata, Mr. Hiroki Oyama, Mr. Kazutoshi Ito and Mr. Yusuke Katagiri for their expertise in performing coronary angioscopy examinations. All authors have approved the submitted version and have agreed both to be personally accountable for the author's own contributions and to ensure that questions related to the accuracy or integrity of any part of the work, even ones in which the author was not personally involved, are appropriately investigated, resolved, and the resolution documented in the literature.
\end{abstract}

\section{Authors' contributions \\ TI made substantial contributions to the conception, design of the work, the acquisition, analysis and interpretation of data, and have drafted the work. YS made substantial contributions to the conception, design of the work, the acquisition and interpretation of data, and have substantively revised it. TT, TK, YH and TO made substantial contributions to the acquisition of data. OI, YS, $\mathrm{YH}$, and TM have substantively revised it. All authors read and approved the final manuscript.}

\section{Funding}

None.

\section{Availability of data and materials}

The datasets used and analysed during the current study are available from the corresponding author on reasonable request.

\section{Ethics approval and consent to participate}

The Medical Ethics Committees of Osaka Police Hospital and Kansai Rosai

Hospital approved this study.

\section{Consent for publication}

All patients provided written informed consent to participate.

\section{Competing interests}

Y. Sotomi received research grants and speaker honoraria from Abbott Vascular Japan, Boston Scientific Japan, TERUMO, Japan Lifeline, Biosensors, and Medtronic, and is an endowed chair funded by TERUMO, Asahi Intecc, NIPRO, and Shimadzu Corporation. O. lida has received remuneration from Medtronic Japan and Boston Scientific Japan. Y. Sakata received Honoraria from Otsuka Pharmaceutical, Daiichi Sankyo, Takeda Pharmaceutical, Mitsubishi Tanabe Pharma, Medtronic Japan and Boehringer Ingelheim Japan, a research grant from Edwards Lifesciences, FUJIFILM RI Pharma, REGiMMUNE, and.Roche Diagnostics, and a Scholarship (educational) grant/endowed chair from Otsuka Pharmaceutical, Johnson \& Johnson, St. Jude Medical Japan, Daiichi Sankyo, Takeda Pharmaceutical, Mitsubishi Tanabe Pharma, Teijin Pharma Limited, Boehringer Ingelheim Japan, Bayer Yakuhin, BIOTRONIK Japan, Boston Scientific, and Medtronic Japan. T. Mano has a research grant from Abbott Vascular Japan. The remaining authors have no disclosures to report.

\section{Author details}

${ }^{1}$ Kansai Rosai Hospital Cardiovascular Center, 3-1-69 Inabaso, Amagasaki 660-8511, Hyogo, Japan. ${ }^{2}$ Department of Cardiovascular Medicine, Osaka University Graduate School of Medicine, Suita, Japan. ${ }^{3}$ Department of Cardiology, Osaka Police Hospital, Osaka, Japan.

Received: 22 August 2020 Accepted: 15 November 2020

Published online: 02 December 2020

\section{References}

1. Stratton IM, Adler Al, Neil HA, Matthews DR, Manley SE, Cull CA, et al. Association of glycaemia with macrovascular and microvascular complications of type 2 diabetes (UKPDS 35): prospective observational study. BMJ. 2000;321:405-12.

2. Norhammar A, Malmberg K, Diderholm E, Lagerqvist B, Lindahl B, Rydén $L$, et al. Diabetes Mellitus: The Major Risk Factor in Unstable Coronary Artery Disease Even After Consideration of the Extent of Coronary Artery Disease and Benefits of Revascularization. J Am Coll Cardiol. 2004;43:585-91.

3. Creager MA, Lüscher TF, Cosentino F, Beckman JA. Diabetes and Vascular Disease: Pathophysiology, Clinical Consequences, and Medical Therapy: Part I. Circulation. 2003;108:1527-32.

4. Valgimigli M, Bueno H, Byrne RA, Collet JP, Costa F, Jeppsson A, et al. 2017 ESC focused update on dual antiplatelet therapy in coronary artery disease developed in collaboration with EACTS: The Task Force for dual antiplatelet therapy in coronary artery disease of the European Society of Cardiology (ESC) and of the European Association for Cardio-Thoracic Surgery (EACTS). Eur Heart J. 2018;39:213-60.

5. Levine GN, Bates ER, Bittl JA, Brindis RG, Fihn SD, Fleisher LA, et al 2016 ACC/AHA Guideline Focused Update on Duration of Dual Antiplatelet Therapy in Patients With Coronary Artery Disease: A Report of the American College of Cardiology/American Heart Association Task Force on Clinical Practice Guidelines: An Update of the 2011 ACCF/AHA/SCAI Guideline for Percutaneous Coronary Intervention, 2011 ACCF/AHA Guideline for Coronary Artery Bypass Graft Surgery, 2012 ACC/AHA/ACP/ AATS/PCNA/SCAI/STS Guideline for the Diagnosis and Management of Patients With Stable Ischemic Heart Disease, 2013 ACCF/AHA Guideline for the Management of ST-Elevation Myocardial Infarction, 2014 AHA/ ACC Guideline for the Management of Patients With Non-ST-Elevation Acute Coronary Syndromes, and 2014 ACC/AHA Guideline on Perioperative Cardiovascular Evaluation and Management of Patients Undergoing Noncardiac Surgery. Circulation. 2016;134:e123-55.

6. Ueda Y, Nanto S, Komamura K, Kodama K. Neointimal coverage of stents in human coronary arteries observed by angioscopy. J Am Coll Cardiol. 1994;23:341-6.

7. Sakai S, Mizuno K, Yokoyama S, Tanabe J, Shinada T, Seimiya K, et al. Morphologic changes in infarct-related plaque after coronary stent placement: a serial angioscopy study. J Am Coll Cardiol. 2003;42:1558-65.

8. Takano M, Mizuno K, Yokoyama S, Tanabe J, Shinada T, Seimiya K, et al. Changes in coronary plaque color and morphology by lipid-lowering therapy with atorvastatin: serial evaluation by coronary angioscopy. J Am Coll Cardiol. 2003;42:680-6. 
9. Ishihara T, Tsujimura T, Okuno S, lida O, Asai M, Masuda M, et al. Early- and middle-phase arterial repair following bioresorbable- and durable-polymer drug-eluting stent implantation: An angioscopic study. Int J Cardiol. 2019;285:27-31.

10. Higo T, Ueda Y, Oyabu J, Okada K, Nishio M, Hirata A, et al. Atherosclerotic and thrombogenic neointima formed over sirolimus drug-eluting stent: an angioscopic study. J Am Coll Cardiol Img. 2009;2:616-24.

11. Ueda Y, Asakura M, Yamaguchi O, Hirayama A, Hori M, Kodama K. The healing process of infarct-related plaques. Insights from 18 months of serial angioscopic follow-up. J Am Coll Cardiol. 2001;38:1916-22.

12. den Heijer P, Foley DP, Hillege HL, Lablanche JM, van Dijk RB, Franzen $D$, et al. The 'Ermenonville' classification of observations at coronary angioscopy-evaluation of intra- and inter-observer agreement. European Working Group on Coronary Angioscopy. Eur Heart J. 1994;15:815-22.

13. Ueda Y, Matsuo K, Nishimoto Y, Sugihara R, Hirata A, Nemoto T, et al. In-Stent Yellow Plaque at 1 Year After Implantation Is Associated With Future Event of Very Late Stent Failure: The DESNOTE Study (Detect the Event of Very late Stent Failure From the Drug-Eluting Stent Not Well Covered by Neointima Determined by Angioscopy). J Am Coll Cardiol Intv. 2015:8:814-21.

14. Sotomi Y, Suzuki S, Kobayashi T, Hamanaka Y, Nakatani S, Hirata A, et al. Impact of the one-year angioscopic findings on long-term clinical events in 504 patients treated with first-generation or second-generation drugeluting stents: the DESNOTE-X study. Eurolntervention. 2019;15:631-9.

15. Haase J, Escaned J, van Swijndregt EM, Ozaki Y, Gronenschild E, Slager CJ, et al. Experimental validation of geometric and densitometric coronary measurements on the new generation Cardiovascular Angiography Analysis System (CAAS II). Cathet Cardiovasc Diagn. 1993;30:104-14.

16. Farb A, Sangiorgi G, Carter AJ, Walley VM, Edwards WD, Schwartz RS, et al. Pathology of acute and chronic coronary stenting in humans. Circulation. 1999;99:44-52

17. Jinnouchi H, Otsuka F, Sato Y, Bhoite RR, Sakamoto A, Torii S, et al. Healthy Strut Coverage After Coronary Stent Implantation: An Ex Vivo Human Autopsy Study. Circ Cardiovasc Interv. 2020;13:e008869.

18. Nakazawa G, Finn AV, Vorpahl M, Ladich ER, Kolodgie FD, Virmani R. Coronary responses and differential mechanisms of late stent thrombosis attributed to first-generation sirolimus- and paclitaxel-eluting stents. $J$ Am Coll Cardiol. 2011;57:390-8.

19. Finn AV, Joner M, Nakazawa G, Kolodgie F, Newell J, John MC, et al. Pathological correlates of late drug-eluting stent thrombosis: strut coverage as a marker of endothelialization. Circulation. 2007;115:2435-41.

20. Taniwaki M, Radu MD, Zaugg S, Amabile N, Garcia-Garcia HM, Yamaji K, et al. Mechanisms of Very Late Drug-Eluting Stent Thrombosis Assessed by Optical Coherence Tomography. Circulation. 2016;133:650-66.

21. Lee SY, Ahn JM, Mintz GS, Hur SH, Choi SY, Kim SW, et al. Characteristics of Earlier Versus Delayed Presentation of Very Late Drug-Eluting Stent Thrombosis: An Optical Coherence Tomographic Study. J Am Heart Assoc. 2017;6:e005386. https://doi.org/10.1161/JAHA.116.005386.

22. Nakamura D, Attizzani GF, Toma C, Sheth T, Wang W, Soud M, et al. Failure Mechanisms and Neoatherosclerosis Patterns in Very Late Drug-Eluting and Bare-Metal Stent Thrombosis. Circ Cardiovasc Interv. 2016;9. pii: e003785.

23. Briguori C, Quintavalle C, Donahue M, D'Alessio F, D'Amore C, Signoriello $\mathrm{G}$, et al. Predictors of strut coverage of drug eluting stent implantation in diabetic patients. Int J Cardiol. 2019;276:61-5.

24. Kubo T, Akasaka T, Tanimoto T, Takano M, Seino Y, Nasu K, et al. Assessment of vascular response after drug-eluting stents implantation in patients with diabetes mellitus: an optical coherence tomography substudy of the J-DESsERT. Heart Vessels. 2016;31:465-73.

25. Kuroda M, Shinke T, Otake H, Sugiyama D, Takaya T, Takahashi H, et al. Effects of daily glucose fluctuations on the healing response to everolimus-eluting stent implantation as assessed using continuous glucose monitoring and optical coherence tomography. Cardiovasc Diabetol. 2016;15:79.

26. Kurihara O, Takano M, Yamamoto M, Shirakabe A, Kimata N, Inami T, et al. Impact of Prediabetic Status on Coronary Atherosclerosis: A Multivessel Angioscopic Study. Diabetes Care. 2013;36:729-33.

27. Kurihara O, Takano M, Mizuno K, Shibata Y, Matsushita M, Komiyama H, et al. Impact of Diabetic Retinopathy on Vulnerability of Atherosclerotic Coronary Plaque and Incidence of Acute Coronary Syndrome. Am J Cardiol. 2016;118:944-9.
28. Kato K, Yonetsu T, Kim SJ, Xing L, Lee H, McNulty I, et al. Comparison of nonculprit coronary plaque characteristics between patients with and without diabetes: a 3-vessel optical coherence tomography study. J Am Coll Cardiol Intv. 2012;5:1150-8.

29. Cook S, Wenaweser P, Togni M, Billinger M, Morger C, Seiler C, et al. Incomplete stent apposition and very late stent thrombosis after drug-eluting stent implantation. Circulation. 2007;115:2426-34.

30. Kim BK, Shin DH, Kim JS, Ko YG, Choi D, Jang Y, et al. Randomized comparison of acute stent malapposition between platinum-chromium versus cobalt-chromium everolimus-eluting stents. Int J Cardiovasc Imaging. 2015;31:269-77.

31. Zhao HQ, Nikanorov A, Virmani R, Jones R, Pacheco E, Schwartz LB. Late stent expansion and neointimal proliferation of oversized Nitinol stents in peripheral arteries. Cardiovasc Intervent Radiol. 2009;32:720-6.

32. Sotomi Y, Tateishi H, Suwannasom P, Dijkstra J, Eggermont J, Liu S, et al. Quantitative assessment of the stent/scaffold strut embedment analysis by optical coherence tomography. Int J Cardiovasc Imaging. 2016;32:871-83.

33. Sotomi Y, Onuma Y, Dijkstra J, Eggermont J, Liu S, Tenekecioglu E, et al. Impact of Implantation Technique and Plaque Morphology on Strut Embedment and Scaffold Expansion of Polylactide Bioresorbable Scaffold - Insights From ABSORB Japan Trial. Circ J. 2016;80:2317-26.

34. Chandrasekhar J, Dangas G, Baber U, Sartori S, Qadeer A, Aquino M, et al. Impact of insulin treated and non-insulin-treated diabetes compared to patients without diabetes on 1-year outcomes following contemporary PCI. Catheter Cardiovasc Interv. 2020;96:298-308.

35. Action to Control Cardiovascular Risk in Diabetes Study Group. Gerstein $\mathrm{HC}$, Miller ME, et al. Effects of intensive glucose lowering in type 2 diabetes. N Engl J Med 2008;358:2545-59.

36. Meinert CL, Knatterud GL, Prout TE, Klimt CR. A study of the effects of hypoglycemic agents on vascular complications in patients with adultonset diabetes. II. Mortality results. Diabetes. 1970;19(Suppl):789-830.

37. Genuth S. Exogenous insulin administration and cardiovascular risk in noninsulin dependent and insulin-dependent diabetes mellitus. Ann Intern Med. 1996;124:104-9.

38. Nusca A, Tuccinardi D, Proscia C, Melfi R, Manfrini S, Nicolucci A, et al. Incremental role of glycaemic variability over HbA1c in identifying type 2 diabetic patients with high platelet reactivity undergoing percutaneous coronary intervention. Cardiovasc Diabetol. 2019;18:147.

39. Lee S, Ay C, Kopp CW, Panzer S, Gremmel T. Impaired glucose metabolism is associated with increased thrombin generation potential in patients undergoing angioplasty and stenting. Cardiovasc Diabetol. 2018;17:131.

40. Awata M, Kotani J, Uematsu M, Morozumi T, Watanabe T, Onishi T, et al. Serial angioscopic evidence of incomplete neointimal coverage after sirolimus-eluting stent implantation: comparison with bare-metal stents. Circulation. 2007;116:910-6.

41. Schwartz RS. Pathophysiology of restenosis: interaction of thrombosis, hyperplasia, and/or remodeling. Am J Cardiol. 1998;81:14E-17E.

42. Yeh RW, Secemsky EA, Kereiakes DJ, Normand SL, Gershlick AH, Cohen DJ, et al. Development and Validation of a Prediction Rule for Benefit and Harm of Dual Antiplatelet Therapy Beyond 1 Year After Percutaneous Coronary Intervention. JAMA. 2016;315:1735-49.

43. Costa F, van Klaveren D, James S, Heg D, Räber L, Feres F, et al. Derivation and validation of the predicting bleeding complications in patients undergoing stent implantation and subsequent dual antiplatelet therapy (PRECISE-DAPT) score: a pooled analysis of individual-patient datasets from clinical trials. Lancet. 2017;389:1025-34.

44. Baber U, Mehran R, Giustino G, Cohen DJ, Henry TD, Sartori S, et al. Coronary Thrombosis and Major Bleeding After PCI With Drug-Eluting Stents Risk Scores From PARIS. J Am Coll Cardiol. 2016;67:2224-34.

45. Bhatt DL, Bonaca MP, Bansilal S, Angiolillo DJ, Cohen M, Storey RF, et al. Reduction in Ischemic Events With Ticagrelor in Diabetic Patients With Prior Myocardial Infarction in PEGASUS-TIMI 54. J Am Coll Cardiol. 2016;67:2732-40.

46. Hahn JY, Song YB, Oh JH, Cho DK, Lee JB, Doh JH, et al. 6-month versus 12 -month or longer dual antiplatelet therapy after percutaneous coronary intervention in patients with acute coronary syndrome (SMART-DATE): a randomised, open-label, non-inferiority trial. Lancet. 2018;391:1274-84.

47. Kedhi E, Fabris E, van der Ent M, Buszman P, von Birgelen C, Roolvink $V$, et al. Six months versus 12 months dual antiplatelet therapy after 
drug-eluting stent implantation in ST-elevation myocardial infarction (DAPT-STEMI): randomised, multicentre, non-inferiority trial. BMJ. 2018;363:k3793.

48. Watanabe H, Domei T, Morimoto T, Natsuaki M, Shiomi H, Toyota T, et al. Effect of 1-Month Dual Antiplatelet Therapy Followed by Clopidogrel vs 12-Month Dual Antiplatelet Therapy on Cardiovascular and Bleeding Events in Patients Receiving PCI: The STOPDAPT-2 Randomized Clinical Trial. JAMA. 2019;321:2414-27.

49. Sharma A, Garg A, Elmariah S, Drachman D, Obiagwu C, Vallakati A, et al. Duration of Dual Antiplatelet Therapy Following Drug-Eluting Stent Implantation in Diabetic and Non-Diabetic Patients: A Systematic Review and Meta-Analysis of Randomized Controlled Trials. Prog Cardiovasc Dis. 2018;60:500-7.

50. Krackhardt F, Waliszewski M, Rischner J, Piot C, Pansieri M, Ruiz-Poveda $\mathrm{FL}$, et al. Nine-month clinical outcomes in patients with diabetes treated with polymer-free sirolimus-eluting stents and 6 month vs. 12 month dual-antiplatelet therapy (DAPT). Herz. 2019;44:433-9.

\section{Publisher's Note}

Springer Nature remains neutral with regard to jurisdictional claims in published maps and institutional affiliations.
Ready to submit your research? Choose BMC and benefit from:

- fast, convenient online submission

- thorough peer review by experienced researchers in your field

- rapid publication on acceptance

- support for research data, including large and complex data types

- gold Open Access which fosters wider collaboration and increased citations

- maximum visibility for your research: over $100 \mathrm{M}$ website views per year

At BMC, research is always in progress.

Learn more biomedcentral.com/submissions 